\title{
GEODESICS IN A QUASI-SPHERICAL SPACETIME: A CASE OF GRAVITATIONAL REPULSION
}

\author{
L. Herrera* \\ Escuela de Física, Facultad de Ciencias, \\ Universidad Central de Venezuela, \\ Caracas, Venezuela.
}

*Postal address: Apartado 80793, Caracas 1080 A, Venezuela; e-mail address: laherrera@telcet.net.ve 


\begin{abstract}
Geodesics are studied in one of the Weyl metrics, referred to as the M-Q solution. First, arguments are provided, supporting our belief that this space-time is the more suitable (among the known solutions of the Weyl family) for discussing the properties of strong quasi-spherical gravitational fields. Then, the behaviour of geodesics is compared with the spherically symmetric situation, bringing out the sensitivity of the trajectories to deviations from spherical symmetry. Particular attention deserves the change of sign in proper radial acceleration of test particles moving radially along symmetry axis, close to the $r=2 M$ surface, and related to the quadrupole moment of the source.
\end{abstract}




\section{Introduction}

As we know, all physical systems in nature (without exception) are submitted to fluctuations, and of course this well established fact also applies to self-gravitating systems. Accordingly we have to accept that any physical property of such a system, e.g. spherical symmetry (if assumed) is to be submitted to such fluctuations. In this paper we shall deal with two obvious questions arising from these general comments, namely:

- How to describe such fluctuations?.

- How sensitive are the properties of compact objects to deviations from spherical symmetry?.

Now, if the field produced by such self-gravitating systems is not particularly intense (the boundary of the source is much larger than the horizon) and the deviation from spherical symmetry is slight, then there is not problem in representing the corresponding deviations from spherical symmetry (both inside and outside the source) as a suitable perturbation of the spherically symmetric exact solution [1] (although strictly speaking the term "horizon" refers to the spherically symmetric case, we shall use it when considering $r=2 M$ surface, in the case of small deviations from sphericity).

However, as the object becomes more and more compact, such perturbative schemes will eventually fail close to the source. Indeed, as is well known [2], though usually overlooked, as the boundary surface of the source approaches the horizon (in the sense indicated above), any finite perturbation of the Schwarzschild spacetime, becomes fundamentally different from any Weyl metric, even if the latter is characterized by parameters whose values are arbitrarily close to those corresponding to spherical symmetry. In other words, for strong gravitational fields, no matter how small are the multipole moments (higher than monopole) of the source, there exists a bifurcation between the perturbed Schwarzschild metric and all the other Weyl metrics. This in turn, is just the expression of the fact [3], that the only static and asymptotically-flat vacuum space-time possessing a regular horizon is the Schwarzschild solution. For all the others Weyl exterior solutions [4], the physical components of the Riemann tensor exhibit singularities at $r=2 M$.

In line with these arguments, let us mention that in recent works [5], [6] it has been shown that such bifurcation between the exactly spherically 
symmetric case and a solution of the Weyl family, when considering the source (the interior), takes place for strong gravitational fields, but before the horizon is reached.

It is worth mentioning that the sensitivity of the trajectories of test particles in the $\gamma$ spacetime [7], to small changes of $\gamma$, for orbits close to $2 M$, has been brought out [8]. Also, the influence of the quadrupole moment on the motion of test particles within the context of Erez-Rosen metric [9] has been investigated by many authors (see [10], [11] and references therein)

Motivated by the above mentioned facts, we want to present in this work another approach to the problem of describing deviations from spherical symmetry. This consists in considering an exact solution of Einstein equations (of the Weyl family, since we restrict ourselves to static axially-symmetric solutions) continuously linked to the Schwarzschild metric, through one of its parameters, instead of considering a perturbation of the Schwarzschild space-time.

However, since there are as many different (physically distinguishable [12]) Weyl solutions as there are different harmonic functions (see next section), then the obvious question arises: which among Weyl solutions is better entitled to describe small deviations from spherical symmetry?.

Although it should be obvious that such question does not have not a unique answer (there is an infinite number of ways of being non-spherical, so to speak), we shall invoke a very simple criterion, emerging from Newtonian gravity, in order to choose our solution.

Indeed, in order to answer the question above, let us recall [13] that most known Weyl solutions, present a serious drawback when describing quasispherical space-times. It consists of the fact that its multipole structure is such that multipole moments, higher than quadrupole, are of the same order as the quadrupole moment. Instead, as it is intuitively clear, the relevance of such multipole moments should decrease as we move from lower to higher moments, the quadrupole moment being the most relevant for a small departure from sphericity. Thus for example in Newtonian gravity, multipole moments of an ellipsoid of rotation, with homogeneous density, and axes (a,a,b) read:

$$
\left\{\begin{array}{l}
D_{2 n}=\frac{(-2)^{n} 3 M a^{2 n}}{(2 n+1)(2 n+3)} \epsilon^{n}(1-\epsilon / 2)^{n}, \quad \epsilon \equiv \frac{a-b}{a} \\
D_{2 n+1}=0
\end{array}\right.
$$


because of the factor $\epsilon^{n}$, this equation clearly exhibits the progressive decreasing of the relevance of multipole moments as $n$ increases.

Thus, in order to describe small departures from sphericity, by means of exact solutions of the Einstein equations, we would require an exact solution whose relativistic multipole structure shares the property mentioned above. Fortunately enough, such a solution exists.

Indeed, there is one (exact) solution of the Weyl family (hereafter referred to as the $\mathrm{M}-\mathrm{Q}$ solution [13]), which may be interpreted as a quadrupole correction to the Schwarzschild space-time (see below). It is for this exterior metric that we are going to study the motion of test particles (more exactly, for a sub-class of this solution).

As we shall see, for very strong fields, arbitrarily small values of the quadrupole moment of the source, introduce important differences in the radial motion of test particles, as compared with the exactly spherically symmetric case. Particularly interesting is the appearance of repulsive forces, related to the quadrupole moment, acting on radially moving particles, along the symmetry axis and close to the horizon.

The paper is organized as follows. In the next section we give a brief resume of Weyl solutions. Next, we describe the $\mathrm{M}-\mathrm{Q}$ solution [13] and comment on its properties, in Section 3. The geodesic equations for the MQ solution are found and analyzed in Section 4. Finally results are discussed in the last Section.

\section{The Weyl metrics}

Static axysymmetric solutions to Einstein equations are given by the Weyl metric [4]

$$
d s^{2}=e^{2 \Psi} d t^{2}-e^{-2 \Psi}\left[e^{2 \Gamma}\left(d \rho^{2}+d z^{2}\right)+\rho^{2} d \phi^{2}\right],
$$

where metric functions have to satisfy

$$
\Psi_{, \rho \rho}+\rho^{-1} \Psi_{, \rho}+\Psi_{, z z}=0
$$

and

$$
\Gamma_{, \rho}=\rho\left(\Psi_{, \rho}^{2}-\Psi_{, z}^{2}\right) \quad ; \quad \Gamma_{, z}=2 \rho \Psi_{, \rho} \Psi_{, z} .
$$

Observe that (2) is just the Laplace equation for $\Psi$ (in the Euclidean space), and furthermore it represents the integrability condition for (3), im- 
plying that for any "Newtonian" potential we have a specific Weyl metric, a well known result.

The general solution of the Laplace equation (2) for the function $\Psi$, presenting an asymptotically flat behaviour, results to be

$$
\Psi=\sum_{n=0}^{\infty} \frac{a_{n}}{r^{n+1}} P_{n}(\cos \theta)
$$

where $r=\left(\rho^{2}+z^{2}\right)^{1 / 2}, \cos \theta=z / r$ are Weyl spherical coordinates and $P_{n}(\cos \theta)$ are Legendre Polynomyals. The coefficients $a_{n}$ are arbitrary real constants which have been named in the literature "Weyl moments", although they cannot be identified as relativistic multipole moments in spite of the formal similarity between expression (4) and the Newtonian potential.

Another interesting way of writting the solution (4) was obtained by ErezRosen [9] and Quevedo [14], integrating equations $(2,3)$ in prolate spheroidal coordinates, which are defined as follows

$$
\begin{aligned}
x & =\frac{r_{+}+r_{-}}{2 \sigma}, \quad y=\frac{r_{+}-r_{-}}{2 \sigma} \\
r_{ \pm} & \equiv\left[\rho^{2}+(z \pm \sigma)^{2}\right]^{1 / 2} \\
x & \geq 1 \quad, \quad-1 \leq y \leq 1,
\end{aligned}
$$

where $\sigma$ is an arbitrary constant which will be identified later with the Schwarzschild's mass. The prolate coordinate $x$ represents a radial coordinate, whereas the other coordinate, $y$ represents the cosine function of the polar angle.

In these prolate spheroidal coordinates, $\Psi$ takes the form

$$
\Psi=\sum_{n=0}^{\infty}(-1)^{n+1} q_{n} Q_{n}(x) P_{n}(y)
$$

being $Q_{n}(y)$ Legendre functions of second kind and $q_{n}$ a set of arbitrary constants. The corresponding expression for the function $\Gamma$, has been obtained by Quevedo [14]

A sub-family of Weyl solutions has been obtained by Gutsunaev and Manko [15],[16] starting from the Schwarzchild solution as a "seed" solution.

Both sets of coefficients, $a_{n}$ and $q_{n}$, characterize any Weyl metric [14]. Nevertheless, as mentioned before, these constants do not give us physical 
information about the metric since they do not represent the "real" multipole moments of the source. That is not the case for the relativistic multipole moments firstly defined by Geroch [17], and subsequently by Hansen [18] and Thorne [19], which, as it is known, characterize completely and uniquely, at least in the neighbourhood of infinity, every asymptotycally flat and stationary vacuum solution [20], providing at the same time a physical description of the corresponding solution.

An algorithm to calculate the Geroch multipole moments was developed by G.Fodor, C. Hoenselaers and Z. Perjes [21] (FHP). By applying such a method, the resulting multipole moments of the solution are expressed in terms of the Weyl moments. Similar results are obtained from Thorne's definition, using harmonic coordinates. The structure of the obtained relation between coefficients $a_{n}$ and these relativistic moments allows to express the Weyl moments as a combination of the Geroch relativistic moments.

\subsection{The Monopole-Quadrupole solution, $M-Q$}

In this section we would like to review the properties of a solution (hereafter referred to as the $M-Q$ solution [13]) which is particularly suitable for the study of perturbations of spherical symmetry. The main argument to support this statement is based on the fact that previously known Weyl metrics (e.g. Gutsunaev-Manko [15], Manko [16], $\gamma$-metric [7], etc.) have a multipolar structure (in the Geroch sense) such that all the moments higher than the quadrupole, are of the same order as the quadrupole moment. In fact for the above mentioned metrics we have (odd moments are of course vanishing)

$$
\begin{aligned}
& M_{0}^{G M}=M_{0}^{E R}=M \\
& M_{2}^{G M}=M_{2}^{E R}=\frac{2}{15} q_{2} M^{3} \\
& M_{4}^{G M}=-3 M_{4}^{E R}=\frac{4}{35} q_{2} M^{5} \\
& M_{6}^{G M}=M_{6}^{E R}-\frac{2}{7} \frac{817}{33} M^{2} M_{4}^{E R}=\frac{2}{15} \frac{4}{231} q_{2} M^{7}\left(\frac{194}{7}+\frac{14}{15} q_{2}\right),
\end{aligned}
$$

where $q_{2}$ is the quadrupole parameter in the Erez-Rosen metric. For the gamma metric the results are

$$
M_{0}=\gamma M
$$




$$
\begin{aligned}
& M_{2}=\gamma \frac{M^{3}}{3}\left(1-\gamma^{2}\right) \\
& M_{4}=\gamma \frac{M^{5}}{5}\left(1-\gamma^{2}\right)\left(1-\frac{19}{21} \gamma^{2}\right) \\
& M_{6}=\gamma \frac{M^{7}}{7}\left(1-\gamma^{2}\right)\left(1+\frac{389}{495} \gamma^{4}-\frac{292}{165} \gamma^{2}\right)
\end{aligned}
$$

For the Curzon metric [22], the situation is still worse since it posseses a unique parameter which represents the mass, and all higher moments are proportional to increasing powers of that parameter, i.e., $M_{0}=-a_{0}, M_{2}=$ $\frac{1}{3} a_{0}^{3}, M_{4}=-\frac{19}{105} a_{0}^{5}, M_{6}=\frac{389}{3465} a_{0}^{7}$.

From the comments above it should be clear that neither of the mentioned metrics satisfies the criterion described in the Introduction.

In [13] it was shown that it is possible to find a solution of the Weyl family, by a convenient choice of coefficients $a_{n}$, such that the resulting solution possesses only monopole and quadrupole moments (in the Geroch sense). The obtained solution $(M-Q)$ may be written as series of GutsunayevManko [15] and Erez-Rosen [9] solutions, as follows:

$$
\Psi_{M-Q}=\Psi_{q^{0}}+q \Psi_{q^{1}}+q^{2} \Psi_{q^{2}}+\ldots=\sum_{\alpha=0}^{\infty} q^{\alpha} \Psi_{q^{\alpha}}
$$

where the zeroth order corresponds to the Schwarzschild solution.

$$
\Psi_{q^{0}}=-\sum_{n=0}^{\infty} \frac{\lambda^{2 n+1}}{2 n+1} P_{2 n}(\cos \theta)
$$

with $\lambda \equiv M / r$ and it appears that each power in $q$ adds a quadrupole correction to the spherically symmetric solution. Now, it should be observed that due to the linearity of Laplace equation, these corrections give rise to a series of exact solutions. In other words, the power series of $q$ may be cut at any order, and the partial summation, up to that order, gives an exact solution representing a quadrupolar correction to the Schwarzschild solution.

The simplest way to interpret physically the exact solutions obtained from the quadrupolar corrections described above, consists in analyzying the corresponding multipolar structure. Thus, it can be shown that cutting solution (8) at some order $\alpha$, one obtains an exact solution with the following properties: 
- Both, the monopole and the quadrupole moments are non-vanishing: $M_{0} \equiv M, M_{2} \equiv q M^{3}$.

- All the moments beyond $M_{2}$, until order $2(\alpha+1)$ (inclusive) vanish.

- All moments above $M_{2(\alpha+1)}$ are of order $q^{\alpha+1}$. Therefore, the solution represents a quadrupolar correction to the Schwarzschild solution, which is an exact solution up to the given order.

Since we are interested in slight deviations from spherical symmetry, we shall consider the $\mathrm{M}-\mathrm{Q}$ solution, only up to first order in $q$. Thus, the explicit solution up to first order, describing a quadrupolar correction to the monopole, is ( note a misprint in the equation (13) in [23])

$$
\begin{aligned}
\Psi_{M-Q}^{(1)} & \equiv \Psi_{q^{0}}+q \Psi_{q^{1}}=\frac{1}{2} \ln \left(\frac{x-1}{x+1}\right)+\frac{5}{8} q\left(3 y^{2}-1\right) \times \\
& \times\left[\left(\frac{3 x^{2}-1}{4}-\frac{1}{3 y^{2}-1}\right) \ln \left(\frac{x-1}{x+1}\right)-\frac{2 x}{\left(x^{2}-y^{2}\right)\left(3 y^{2}-1\right)}+\frac{3 x}{2}\right](10) \\
\Gamma_{M-Q}^{(1)} & \equiv \Gamma_{q^{0}}+q \Gamma_{q^{1}}+q^{2} \Gamma_{q^{2}}=\frac{1}{2}\left(1+\frac{225}{24} q^{2}\right) \ln \left(\frac{x^{2}-1}{x^{2}-y^{2}}\right) \\
& -\frac{15}{8} q x\left(1-y^{2}\right)\left[1-\frac{15}{32} q\left(x^{2}+7 y^{2}-9 x^{2} y^{2}+1-\frac{8}{3} \frac{x^{2}+1}{x^{2}-y^{2}}\right)\right] \ln \left(\frac{x-1}{x+1}\right) \\
& +\frac{225}{1024} q^{2}\left(x^{2}-1\right)\left(1-y^{2}\right)\left(x^{2}+y^{2}-9 x^{2} y^{2}-1\right) \ln 2\left(\frac{x-1}{x+1}\right) \\
& -\frac{15}{4} q\left(1-y^{2}\right)\left[1-\frac{15}{64} q\left(x^{2}+4 y^{2}-9 x^{2} y^{2}+4\right)\right] \\
& -\frac{75}{16} q^{2} x^{2} \frac{1-y^{2}}{x^{2}-y^{2}}-\frac{5}{4} q\left(x^{2}+y^{2}\right) \frac{1-y^{2}}{\left(x^{2}-y^{2}\right)^{2}} \\
& -\frac{75}{192} q^{2}\left(2 x^{6}-x^{4}+3 x^{4} y^{2}-6 x^{2} y^{2}+4 x^{2} y^{4}-y^{4}-y^{6}\right) \frac{1-y^{2}}{\left(x^{2}-y^{2}\right)^{4}}
\end{aligned}
$$

The first twelve Geroch moments of this solution are (odd moments vanish, because of the reflection symmetry)

$$
M_{0}=M, M_{2}=M^{3} q, M_{4}=0, M_{6}=-\frac{60}{77} M^{7} q^{2}
$$




$$
\begin{aligned}
M_{8} & =-\frac{1060}{3003} M^{9} q^{2}-\frac{40}{143} M^{9} q^{3}, M_{10}=-\frac{19880}{138567} M^{11} q^{2}+\frac{146500}{323323} M^{11} q^{3} \\
M_{12} & =-\frac{23600}{437437} M^{13} q^{2}+\frac{517600}{1062347} M^{13} q^{3}+\frac{4259400}{7436429} M^{13} q^{4}
\end{aligned}
$$

From the expressions above, it is apparent that the parameter $q \equiv M_{2} / M^{3}$ representing the quadrupole moment, enters into the multipole moments $M_{2 n}$, for $n \geq 2$, only at order 2 or higher. Accordingly, solution $(10,11)$ for a small value of $q$, up to order $q$, may be interpreted as the gravitational field outside a quasi-spherical source. The spacetime being represented by a quadrupole correction to the monopole (Schwarzschild) solution. This is in contrast with other previously mentioned solutions of Weyl family, where all moments higher than the quadrupole are of the same order in $q$ as the quadrupole. And therefore for small values of $q$ they cannot be interpreted as a quadrupole perturbation of spherical symmetry.

Instead of cylindrical corrdinates $(\rho, z)$, it will be useful for the next section to work with Erez-Rosen coordinates $(r, \theta)$ given by:

$$
\begin{aligned}
z & =(r-M) \cos \theta \\
\rho & =\left(r^{2}-2 M r\right)^{1 / 2} \sin \theta
\end{aligned}
$$

and related to prolate coordinates, by

$$
\begin{aligned}
x & =\frac{r}{M}-1 \\
y & =\cos \theta
\end{aligned}
$$

The metric functions of the solution, up to the first order in $q$, hereafter referred as $\mathrm{M}-\mathrm{Q}^{(1)}$ are:

$$
\begin{aligned}
\Psi_{M-Q}^{(1)} & =\frac{1}{2} \ln \left(1-\frac{2}{R}\right)+\frac{5}{32} q\left(3 y^{2}-1\right)\left(3 R^{2}-6 R+2\right) \ln \left(1-\frac{2}{R}\right) \\
& -\frac{5}{8} q \ln \left(1-\frac{2}{R}\right)-\frac{5}{4} q \frac{R-1}{(R-1)^{2}-y^{2}}+\frac{15}{16} q\left(3 y^{2}-1\right)(R-1) \\
\Gamma_{M-Q}^{(1)} & =\frac{1}{2} \ln \left[\frac{(R-1)^{2}-1}{(R-1)^{2}-y^{2}}\right]-\frac{15}{8} q\left(1-y^{2}\right)(R-1) \ln \left(1-\frac{2}{R}\right) \\
& -\frac{15}{4} q\left(1-y^{2}\right)-\frac{5}{4} q\left(1-y^{2}\right)\left[\frac{(R-1)^{2}+y^{2}}{\left((R-1)^{2}-y^{2}\right)^{2}}\right]
\end{aligned}
$$


with $R \equiv r / M$.

It is worth noting that the $\mathrm{M}-\mathrm{Q}$ solution (8) up to order $q$, implies the appearance of terms of order $q^{2}$ in $\Gamma_{M-Q}^{(1)}$ as shown in (11). However such terms have not been included in (16) since they do not play any role in the discussion below. Indeed, in the case $y=1$ (along the symmetry axis) all these terms vanish, and in the case $y \neq 1$, in the limit $R->2$, the leading term among the factors multiplying $q^{2}$, is of the same order of magnitude as that multiplying $q$, therefore since we are considering small values of $q$, all terms with $q^{2}$ may be neglected.

In the next section we shall analyze the motion of a test particle in a space-time described by the M-Q(1) solution.

\section{The geodesics for the $\mathrm{M}-\mathrm{Q}^{(1)}$ metric}

The equations governing the geodesics can be derived from the Lagrangian

$$
2 \mathcal{L}=g_{\alpha \beta} \dot{x}^{\alpha} \dot{x}^{\beta},
$$

where the dot denotes differentiation with respect to an affine parameter $s$, which for timelike geodesics coincides with the proper time. Then, from the Euler-Lagrange equations,

$$
\frac{d}{d s}\left(\frac{\partial \mathcal{L}}{\partial \dot{x}^{\alpha}}\right)-\frac{\partial \mathcal{L}}{\partial x^{\alpha}}=0,
$$

it follows:

$$
\begin{gathered}
\ddot{t} g_{t t}+\dot{t}\left(\dot{r} g_{t t, r}+g_{t t, \theta} \dot{\theta}\right)=0 \\
2 \ddot{r} g_{r r}+2 \dot{r}\left(\dot{r} g_{r r, r}+g_{r r, \theta} \dot{\theta}\right)-\dot{t}^{2} g_{t t, r}-\dot{r}^{2} g_{r r, r}-\dot{\theta}^{2} g_{\theta \theta, r}-\dot{\phi}^{2} g_{\phi \phi, r}=0 \\
2 \ddot{\theta} g_{\theta \theta}+2 \dot{\theta}\left(\dot{r} g_{\theta \theta, r}+g_{\theta \theta, \theta} \dot{\theta}\right)-\dot{t}^{2} g_{t t, \theta}-\dot{r}^{2} g_{r r, \theta}-\dot{\theta}^{2} g_{\theta \theta, \theta}-\dot{\phi}^{2} g_{\phi \phi, \theta}=0 \\
\ddot{\phi} g_{\phi \phi}+\dot{\phi}\left(\dot{r} g_{\phi \phi, r}+g_{\phi \phi, \theta} \dot{\theta}\right)=0
\end{gathered}
$$

Where, our exterior metric in Erez-Rosen coordinates read:

$$
\begin{aligned}
& g_{r r}=-e^{2 \Gamma-2 \Psi}\left(1+\frac{\lambda^{2} \sin ^{2} \theta}{1-2 \lambda}\right)=-e^{2 q\left(\Gamma_{q^{1}}-\Psi_{q^{1}}\right)} \frac{1}{\left(1-\frac{2 M}{r}\right)} \\
& g_{\theta \theta}=-e^{2 \Gamma-2 \Psi} r^{2}\left(1-2 \lambda+\lambda^{2} \sin ^{2} \theta\right)=-r^{2} e^{2 q\left(\Gamma_{q^{1}}-\Psi_{q^{1}}\right)}
\end{aligned}
$$




$$
\begin{aligned}
g_{\phi \phi} & =-e^{-2 \Psi} r^{2} \sin ^{2} \theta(1-2 \lambda)=-r^{2} \sin ^{2} \theta e^{-2 q \Psi_{q^{1}}} \\
g_{t t} & =e^{2 \Psi}=\left(1-\frac{2 M}{r}\right) e^{2 q \Psi_{q^{1}}}
\end{aligned}
$$

and the functions $\Gamma_{q^{1}}$ and $\Psi_{q^{1}}$ are given by

$$
\begin{aligned}
\Psi_{q^{1}}= & \frac{5}{32}\left(3 y^{2}-1\right)\left(3 R^{2}-6 R+2\right) \ln \left(1-\frac{2}{R}\right) \\
- & \frac{5}{8} \ln \left(1-\frac{2}{R}\right)-\frac{5}{4} \frac{R-1}{(R-1)^{2}-y^{2}}+\frac{15}{16}\left(3 y^{2}-1\right)(R-1) \\
\Gamma_{q^{1}} & =-\frac{15}{8}\left(1-y^{2}\right)(R-1) \ln \left(1-\frac{2}{R}\right) \\
& -\frac{15}{4}\left(1-y^{2}\right)-\frac{5}{4}\left(1-y^{2}\right)\left[\frac{(R-1)^{2}+y^{2}}{\left((R-1)^{2}-y^{2}\right)^{2}}\right]
\end{aligned}
$$

Since we are concerned only with timelike geodesics, the range of our coordinates is:

$$
\infty>t \geq 0 \quad r>2 M \quad \pi \geq \theta \geq 0 \quad 2 \pi \geq \phi \geq 0
$$

\subsection{Radial geodesics}

Let us consider the motion of a test particle along a radial geodesic, for an arbitrary value of $\theta$. Thus putting $\dot{\theta}=\dot{\phi}=0$ in (20), we obtain

$$
2 \ddot{r} g_{r r}+\dot{r}^{2}\left(g_{r r, r}+\frac{g_{r r} g_{t t, r}}{g_{t t}}\right)-\frac{g_{t t, r}}{g_{t t}}=0
$$

or using (23)

$$
\ddot{r}=-e^{2 q\left(\Psi_{q^{1}}-\Gamma_{q^{1}}\right)}\left(\frac{M}{r^{2}}+\frac{q(R-2)}{R} \Psi_{q^{1}, r}\right)-\dot{r}^{2} q \Gamma_{q^{1}, r}
$$

with $\Psi_{q^{1}}, \Gamma_{q^{1}}, \Psi_{q^{1}, r}$ and $\Gamma_{q^{1}, r}$ easily obtained from $(24,25)$.

In the spherically symmetric case $(q=0)$ we obtain from $(27)$ the well known result

$$
\ddot{r}=-\frac{M}{r^{2}}
$$


Now, before proceeding farther, and in order to express our results in terms of physically meaningful quantitites, let us introduce (locally defined) coordinates associated with a locally Minkowskian observer (or alternatively a tetrad field associated with such Minkowskian observer). Thus, let

$$
d X=\sqrt{-g_{r r}} d r
$$

and

$$
d T=\sqrt{g_{t t}} d t
$$

from where it follows that

$$
\dot{r}=\frac{\frac{d X}{d T}}{\sqrt{g_{r r}\left[\left(\frac{d X}{d T}\right)^{2}-1\right]}}
$$

and

$$
\frac{d^{2} X}{d T^{2}}=\ddot{r} \sqrt{-g_{r r}}\left[1-\left(\frac{d X}{d T}\right)^{2}\right]^{2}-\left(\frac{d X}{d T}\right)^{2} \frac{g_{r r, r}\left[1-\left(\frac{d X}{d T}\right)^{2}\right]}{2\left(-g_{r r}\right)^{(3 / 2)}}
$$

In the spherically symmetric case $(q=0),(32)$ reduces to

$$
\frac{d^{2} X}{d T^{2}}=-\frac{M}{r^{2}}\left[1-\left(\frac{d X}{d T}\right)^{2}\right]\left(1-\frac{2 M}{r}\right)^{-1 / 2}
$$

or, in terms of $R$

$$
\frac{d^{2} X}{d T^{2}}=-\frac{R_{, r}}{R^{3 / 2}}\left[1-\left(\frac{d X}{d T}\right)^{2}\right](R-2)^{-1 / 2}
$$

which is also a known result [24]. Since $\left(\frac{d X}{d T}\right)$ is always smaller than one, the attractive nature of gravity for any value of $r$ (larger than $2 M$ ) is clearly exhibited in (33).

Let us now see what is the situation for any value of $q$, arbitrarily small but different from zero, as we approach the horizon. We shall analyze two different situations, namely:

- The particle moves along a radial geodesics, outside the symmetry axis $\left(y^{2} \neq 1\right)$

- The particle moves along the symmetry axis $\left(y^{2}=1\right)$ 


\subsection{1 $y^{2} \neq 1$}

Let us assume that the test particle moves along a radial geodesics outside the symmetry axis, then as $R->2$, the leading term in (32), for small values of $q$ is

$$
\frac{d^{2} X}{d T^{2}} \longrightarrow \frac{-R, r\left[1-\left(\frac{d X}{d T}\right)^{2}\right]}{2^{\frac{3}{2}}(R-2)^{1 / 2}}+\frac{\mathcal{O}(q)}{(R-2)^{1 / 2}}
$$

Observe that this limit (except for small corrections of order $q$ ) is the same as the one obtained in the exactly spherically symmetric situation from (34). Thus for particles moving along radial geodesics (excluding the symmetry axis), small values of the quadrupole moment introduce small perturbations on the trajectories, and the attractive nature of the gravitational force is preserved at all times. Let us now see how this situation change for particles moving along the symmetry axis $\left(y^{2}=1\right)$.

\subsection{2 $\quad y^{2}=1$}

In this case, it follows that as $R->2$,

$$
\ddot{r} \longrightarrow-q \frac{5}{16} \frac{R, r}{(R-2)} e^{-\frac{5 q}{4(R-2)}},
$$

which is of course a positive quantity if $q<0$, and:

$$
\sqrt{-g_{r r}} \longrightarrow \frac{\sqrt{2}}{\sqrt{R-2}} e^{\frac{5 q}{8(R-2)}}
$$

and

$$
g_{r r, r} \longrightarrow \frac{5 q R, r}{2(R-2)^{3}} e^{\frac{5 q}{4(R-2)}}
$$

Using (36-38) in (32), we finally find that as $R->2$

$$
\frac{d^{2} X}{d T^{2}} \longrightarrow \frac{-5 q R, r}{2^{\frac{7}{2}}(R-2)^{3 / 2}}\left[1-\left(\frac{d X}{d T}\right)^{2}\right] e^{\frac{-5 q}{8(R-2)}}
$$

which is a positive quantity if $q<0$, diverging at the horizon, for any, different from zero, value of $q$. Thus, small deviations form spherical symmetry (with $q<0$ ) produce, close to the horizon, a positive (in the sense of increasing $X$ ) radial acceleration of particles moving radially on the symmetry axis, 
as measured by a Lorentzian observer. Therefore, under such circumstances, such observer would infer the existence of a "repulsive" gravitational force acting on the particle. This conclusion is valid in all locally Minkowskian frames, including of course the instantaneous rest frame where $\frac{d X}{d T}=0$. Thus for example, if a test particle,initially at rest with respect to the Minkowskian local frame, is placed close to the horizon (on the axis of symmetry), as seen by such observer, it will tend to displace in the sense of increasing $X$, moving away from the source.

If $q>0$ then $\frac{d^{2} X}{d T^{2}}$ will be negative and tending to zero as $r$ approaches $2 M$.

It is worth noting that the source of this difference between the $y^{2}=1$ and the $y^{2} \neq 1$ cases is the the term $\Psi_{q^{1}, r}$. Indeed, in the later case $\left(y^{2} \neq 1\right)$ we have, as $R->2$,

$$
\Psi_{q^{1}, r} \longrightarrow-\frac{15 R, r\left(1-y^{2}\right)}{16(R-2)},
$$

whereas in the case $y^{2}=1$, we have

$$
\Psi_{q^{1}, r} \longrightarrow \frac{5 R,_{r}}{8(R-2)^{2}},
$$

It should be emphasized, once again, that the results above (36-39), hold for any small, but finite value of $q$. Therefore one should not expect to

obtain the spherically symmmetric limit (34) from (39) as $q \rightarrow 0$, since the spherically symmmetric contribution (in the limit $R \rightarrow 2$ ) being smaller, has been omitted in (39).

Finally, observe that the fulfilment of equation (21), imposes a constraint on $\dot{r}$ which does not play any role in the very appearance of the repulsive effect as implied by equation (39) since, as already mentioned, such an effect is observed by all locally Minkowskian observers, including the instantaneously at rest observer.

\subsection{Circular geodesics}

Let us now consider circular geodesics. From (19)-(22), and the definition of the angular velocity of a test particle along circular geodesics

$$
\omega=\frac{\dot{\phi}}{\dot{t}}
$$


we obtain using $\dot{r}=\dot{\theta}=0$, in the equatorial plane $(\theta=\pi / 2)$

$$
\omega^{2}=\frac{e^{4 q \Psi_{q^{1}}}\left[R,_{r}+R q \Psi_{q^{1}, r}(R-2)\right]}{M^{2} R^{3}\left(R,,_{r}-q R \Psi_{q^{1}, r}\right)}
$$

In the spherically symmetric case $(q=0)$, (43) yields the Kepler law

$$
\omega^{2}=\frac{M}{r^{3}}
$$

However, for arbitrarily small (different from zero) quadrupole moment, (43) exhibits a bifurcation from Kepler law, as $R->2$. Nevertheless, such bifurcation takes place for orbits for which the tangential velocity of the particle is larger than 1, and therefore are deprived of physical interest (see below). Indeed, we have for the tangential velocity $W^{\mu}$ of the test particle along the circular geodesic (see for example [25])

$$
W^{\alpha}=\frac{d \phi}{\sqrt{g_{t t}} d t} \delta_{\phi}^{\alpha}
$$

Then, we obtain on the equatorial plane

$$
W^{2}=\frac{R,,_{r}+R q \Psi_{q^{1}, r}(R-2)}{(R-2)\left(R,_{r}-R q \Psi_{q^{1}, r}\right)}
$$

It follows from (46) that photonic orbits $(W=1)$ differ from $r=3 M(R=$ 3 ) by factors of order $q$. Also, if $R->2$, then $W^{2}->\frac{8}{15 q}$. Of course these orbits are clearly unphysical, being $W>1$.

Finally it is worth noting that circular geodesics on the equatorial plane are stable. Indeed, it follows from (21) with $\dot{r}=\dot{\theta}=0$ that

$$
2 \ddot{\theta} g_{\theta \theta}=\dot{t}^{2}\left(g_{t t, \theta}+\omega^{2} g_{\phi \phi, \theta}\right)
$$

implying $\ddot{\theta}=0$ on the equatorial plane.

\section{Conclusions}

We have first argued that the $\mathrm{M}-\mathrm{Q}^{(1)}$ solution represents a good candidate among known Weyl solutions, to describe small deviations from spherical 
symmetry. Then we have calculated the geodesic equations for a test particle moving in that space-time.

We have found that in general the behaviour of circular geodesics is not very different from the spherically symmetric case. However important differences apppear in the behaviour of radial geodesics. Thus, it has been established that particles along the axis of symmetry, close to $R=2$ feel a repulsive force if $q<0$ (corresponding to an oblate source). It should be obvious that such an effect is absent in Newtonian theory, for small $(q<<1)$ values of the quadrupole moment.

It goes without saying that we have not obtained the full description of the trajectories of test particles in the $\mathrm{M}-\mathrm{Q}^{(1)}$ spacetime, since we have not completely integrated the geodesic equations. Certainly such integration would be necessary in order to extract definitive conclusions about the behaviour of the test particle. Unfortunately we were not able to find analytical solutions to this problem, and a numerical approach is out of the scope of this paper.

It is worth mentioning that repulsives forces in the context of general relativity have been brought out before, the best known example probably being the case in the Reissner-Nordstrom solution (see for example [26]). Also, they appear in connection with rotating and/or unbounded sources [27] or as spurious phenomena associated with coordinate effects (see [24] and references therein).

As an example of the later is the bouncing back of particles that strike the surface $\mathrm{r}=2 \mathrm{M}$, found by Rosen [28] for the spherically symmetric case. However observe that the proper radial acceleration as measured by a locally Minkowskian observer, for the spherically symmetric case, does not exhibit a change of sign in the limit $r->2 M$, as indicated by (33).

In our case, the repulsive character of the force (understood as a change of sign in $\frac{d^{2} X}{d T^{2}}$ ) is exhibited by means of a quantity measured by locally Minkowskian observers, and therefore it is not a coordinate effect, and takes place close to, but before reaching the surface $r=2 M$. In this sense the following comment is in order: It should be clear that at $R=2$ there are not locally Minkowskian observers, since that is a singular surface for any nonvanishing value of $q[11]$. However, for sufficiently small (but finite) values of $q$, close to but at a "finite" distance from $2 M$ (where locally Minkowskian observers do exist) the leading term in $\frac{d^{2} X}{d T^{2}}$ is given by (39). In other words it is in principle possible to find a region where locally Minkowkian observers 
exist and $\frac{d^{2} X}{d T^{2}}$ change of sign.

Finally it is worth asking what are the physical implications of the study presented here. In view of the above, we would say that any result implying the presence of strong gravitational fields and spherical symmetry, should be tested against small fluctuations of such symmetry. Also, it remains to be seen which, if any, might be the implications of the reported repulsive effect, on some astrophysical scenarios, as for example in relativistic jets [29].

\section{References}

[1] B.Boisseau, P.Letelier, Gen.Rel.Grav. 34,1077 (2002).

[2] J. Winicour, A.I. Janis and E.T. Newman, Phys. Rev. 176,1507 (1968); A. Janis, E.T Newman and J. Winicour,Phys. Rev. Lett. 20, 878 (1968); F.I. Cooperstock and G.J. Junevicus Nuov. Cim. 16B, 387 (1973); L. Bel, , Gen. Relativ. Gravitation 1, 337 (1971).

[3] W. Israel, Phys. Rev. 164, 1776 (1967).

[4] H. Weyl,Ann. Phys. (Leipzig), 54, 117 (1917); H. Weyl, Ann. Phys. (Leipzig), 59, 185 (1919); T. Levi.Civita,Atti. Accad. Naz. Lincei Rend. Classe Sci.Fis. Mat. e Nat., 28, 101 (1919); J.L. Synge, Relativity, the general theory (North-Holland Publ. Co, Amsterdam), (1960); D. Kramer, H. Stephani, M.A.H. MacCallum, and E. Herlt, Exact Solutions of Einstein's Field Equations (Cambridge University Press, Cambridge) (1980).

[5] L. Herrera, A. Di Prisco and J. Martinez, Astr. Space Sci. 277, 447 (2001).

[6] L. Herrera, A. Di Prisco and E. Fuenmayor, Class. Quantum Grav. 20, 1125 (2003).

[7] R. Bach and H. Weyl, Math. Z., 13, 134 (1920); G. Darmois, Les equations de la Gravitation Einsteinienne (Gauthier-Villars, Paris) P.36, (1927); D.M. Zipoy,J. Math. Phys., 7, 1137 (1966); R. Gautreau and J.L. Anderson, Phys. Lett., 25A, 291 (1967); F.I. Cooperstock and G.J. Junevicus, Int. J. Theor. Phys., 9, 59 (1968); B.H. Vorhees, Phys. Rev. 
D, 2, 2119 (1970);F. Espósito and L. Witten, Phys. Lett., 58B, 357 (1975); K.S. Virbhadra, Directional naked singularity in General Relativity, preprint gr-qc/9606004.

[8] L. Herrera, F. Paiva and N. O. Santos, Int. J. Modern Phys.D 9, 649 (2000).

[9] G. Erez and N. Rosen,Bull. Res. Council Israel, 8F, 47, (1959).

[10] Ya. Zeldovich and I.D. Novikov, Relativistic Astrophysics (University of Chicago Press, Chicago) (1971); A. Armenti and P. Havas, Relativity and Gravitation Eds. C. Kupper and A. Peres, (Gordon and Breach, London) (1971); H. Quevedo, Fortschr. Phys., 38, 733 (1990).

[11] B. Mashhoon and H. Quevedo, Nuov. Cim., 110B, 291 (1995).

[12] L. Herrera and J.L. Hernández-Pastora, J. Math. Phys. 41, 7544 (2000).

[13] J.L. Hernández-Pastora and J. Martín, Gen.Rel.Grav., 26, 877 (1994).

[14] H. Quevedo, Phys. Rev., 33, 334 (1986).

[15] Gutsunayev, Ts.I., Manko,V.S. Gen.Rel.Grav. 17, 1025 (1985).

[16] Manko, V.S., Gen. Rel. Grav., 21, 1193 (1989).

[17] R. Geroch, J. Math. Phys., 11, 1955 (1970); R. Geroch,J. Math. Phys., 11, 2580 (1970); R. Geroch, J. Math. Phys., 12, 918 (1971).

[18] R. O. Hansen, J. Math. Phys., 15, 46 (1974).

[19] K. S. Thorne, Rev. Mod. Phys., 52, 299 (1980).

[20] R. Beig and W. Simon, Commun. Math. Phys., 78, 75 (1980); R. Beig and W. Simon, Proc. Roy. London., A 376, 333 (1981); P. Kundu,J. Math. Phys., 22, 1236 (1981); ibid 22, 2006 (1981).

[21] G. Fodor, C. Hoenselaers and Z. Perjés, J. Math. Phys., 30, 2252 (1989).

[22] H.E.J. Curzon, Proc. London Math. Soc., 23, 477 (1924). 
[23] J.L. Hernández-Pastora and J. Martín,Class. Quantum Grav., 10, 2581 (1993).

[24] Ch. McGruder, Phys. Rev. D, 25, 3191 (1982).

[25] J. L. Anderson Principles of Relativity (Academic Press, New York,1967).

[26] A. Papapetrou Lectures on General Relativity (D. Reidel, DordrechtHolland, 1974); C. Barbachoux, J. Gariel, G. Marcilhacy and N.O. Santos,Int.J.Modern Phys.D,11, 1255, (2002).

[27] W. B. Bonnor,J. Phys. A10, 1673 (1977); E. L. Schuking, Atti del Convegno sulla Cosmologia (G Barbera Editor, Florence,1966); L. Herrera and N. O. Santos J. Math. Phys. 39, 3817 (1998); W.B. Bonnor, Class. Quantum Grav. 19,146 (2002).

[28] N. Rosen in Relativity (Eds. M. Carmeli, S. Fickler and L. Witten, Plenum Press, New York, 1970).

[29] C. Chicone, B. Mashhoon and B. Punsly Preprint astro-ph/0308421; C. Chicone and B. Mashhoon Preprint astro-ph/0404170 